\title{
Analysis and Experimental Evaluation of Power Line Transmission Parameters for Power Line Communication
}

\author{
P. Mlynek, J. Misurec, M. Koutny, R. Fujdiak, T. Jedlicka \\ Department of Telecommunications, Faculty of Electrical Engineering and Communication, Brno University of Technology, \\ Technicka 12,612 00 Brno, Czech Republic, mlynek@feec.vutbr.cz

\begin{abstract}
The article describes a way of evaluating the power line channel frequency response and input impedance by means of the linear This is followed by measurement evaluation and numerical verification on a simple topology.
\end{abstract} \\ time-invariant (LTI) power line generator. Two possible methods are introduced for the calculation of primary parameters: the \\ first method depends on the physical realization and physical dimension of the cable, and the second method is derived from the \\ data provided by typical electrical cable manufacturers. Based on these methods, a comparison of transfer functions was made.
}

Keywords: Power line communication, primary parameters, time-invariant system, transfer function, simulation, measurement.

\section{INTRODUCTION}

$\mathrm{T}$ HE IDEA of exploiting power distribution networks for data transmission is not new. In certain forms, power lines were used for data transmission almost from the very beginning of building power distribution networks. This was primarily the case of narrowband telephony [1], and later on ripple control or commanding as part of the management of power networks [2].

The nature of power lines, the organization of the distribution network and its management differ from the situation of classical telecommunication or data networks. The power distribution network is primarily designed for the supply of electric power; there are elements in the network which are highly specific for power engineering and not for telecommunications. The data communication between power network nodes has been built with other technologies. Today, the need is rapidly increasing for data transmission, in particular at the low voltage (LV) levels of the so-called "last mile".

Systems for communication over power lines are referred to as PLC (Power Line Communication). PLC systems do not require any specific cabling because the terminal equipment is connected directly to the power network, usually at the LV level $(0.4 \mathrm{kV})$ or, exceptionally, at the HV level $(22 \mathrm{kV})$. PLC thus reduces the operational costs and expenditure for the realization of new telecommunication networks. The cost of realization and installation of a new network is very often more than $50 \%$ of the investment.

PLC systems fall into three areas [3]:

- Ultra Narrow Band (UNB) operating at a very low data rate $(100 \mathrm{bps})$ in the low frequency band. UNB uses one-way communication, used in particular for load control. UNB has a very large operational range (hundreds of kilometres).

- Narrow Band (NB) operating in CENELEC band 3 $148.5 \mathrm{kHz}$ in Europe. Single carrier NB technologies achieve data rates of a few kbps. Nowadays, multicarrier technologies are frequently used which are capable of data rates up to $500 \mathrm{kbps}$. PRIME, G3-PLC, G.hnem and IEEE 1901.2 are the typical examples.
- Broadband operating in the high frequency bands 1.8 $250 \mathrm{MHz}$ has data rates of several megabits per second up to hundreds of Mbps. The typical examples of broadband PLC are devices conforming to the standards TIA-1113 (HomePlug 1.0), IEEE 1901 and ITU-T G.hn.

The analysis and experimental evaluation of power line transmission parameters in this article are conducted especially for the broadband PLC issue. Broadband PLC was initially intended for Internet access applications and successively for Home Area Networking (HAN) applications. A multiyear project funded by the European Community (The Open PLC European Research Alliance, OPERA [4]) inspired most of the recent research efforts in the field of broadband PLC for Internet access. OPERA gave disappointing results in using PLC for Internet access applications. Despite this drawback, the interest of industry came to be focused on in-home application and home networking technology. In these days, broadband PLC has advantages for Smart Home, especially for home networks for complementing WiFi. The main advantage of PLC is the cost saving for the realization of new infrastructure.

On the other hand, there are still several problematic areas in data transmission over power lines. The power line channel is a very harsh and noisy transmission medium. Many factors influence the communication path and path losses. These factors include the cable type, loading, impedances, weather conditions, etc. The article therefore focuses on investigating these factors via simulations and measurements, in particular on the cable type, primary and secondary parameters of the cables and loading impedances or input impedance.

The issue of channel modelling is a key aspect of PLC communication system design and this design must fit the particular characteristic of the power line channel. Thus, the article focuses on the analysis of power line transmission parameters.

Channel impedance is an important parameter in designing the PLC modem. The modem output impedance and the power line input impedance should be matched for 
maximum power transfer between the output of the PLC modem and the power line. Therefore, for an appropriate modem design, the power line impedance must be known and for that reason the input impedance, characteristic impedance, and impedance of branch termination were investigated in this article.

The main contribution of this article is that it provides a comparison of our LTI power line generator and the publicly available simulators in order to investigate parameters which significantly influence the transfer function of the power line channel. The results were also verified by measurements.

\section{POWER LINE CHANNEL MODELLING}

Among many publications in the last decade, the most promising approaches for power line channel modelling were: multipath model [5], two-port network model [6], linear periodically time-variant system [7], time-invariant system [5], and hybrid model based on combining the previous approaches [8].

When the non-linearities of appliance loads are neglected, impedance appliance loads do not vary and the noise is considered stationary, the indoor power line can be considered to be a time-invariant system (LTI). The LTI model is considered because of its simplicity and the channel is considered as a periodical series of channels.

When the non-linearities of appliances cannot be neglected, the indoor power line can be considered a linear periodically time-variant system (LPTV).

\section{LTI POWER LINE GENERATOR}

The LTI power line generator and an analysis with this generator were introduced in [9]. The LTI power line generator was extended by the option of a different calculation of primary parameters, terminal load analysis from measurements, simulations with frequencies up to 100 $\mathrm{MHz}$, input impedance analysis and evaluation of limit values.

In the article, the extended LTI power line generator was used for the investigation of the influence of different approaches to primary parameter calculation on the transfer function.

\section{PLC SIMULATORS BY OTHER AUTHORS}

There already exist two PLC simulators realized in the MATLAB, namely the FTW PLC simulator [10], [11] and the Cañete PLC simulator [12].

The Cañete PLC simulator is based on the bottom-up (deterministic) model. The model is valid in the frequency band up to $30 \mathrm{MHz}$. An advantage of this simulator is that it considers the LTI and LPTV systems.

The FTW PLC simulator is based on the bottom-up frequency-domain model and a realistic description of the topology. It is an open-source simulator with a very detailed documentation.

In comparison with our LTI power line generator described in [9], the simulators by other authors provide similar configurations:
- Cable types with different cross-section $(1.5 ; 2.5 ; 4$; 6 and $10 \mathrm{~mm}^{2}$ ).

- The possibility of divided topology in a particular section.

- Possible configuration of the section length.

- Configuration of terminal impedances.

- Random and fixed topologies.

- LPTV approach [13].

The idea is to compare the FTW PLC simulator and Cañete PLC simulator with our extended LTI power line generator and carry out an analysis of the parameters which most significantly influence the power line communication and in particular the transfer function. The Cañete PLC simulator was considered only in some simulations, because it is not an open source simulator and cannot be modified for all types of simulations.

\section{CALCULATION APPROACHES FOR PRIMARY AND SECONDARY PARAMETERS}

The influence of different approaches to the calculation of primary parameters on the transfer function was also investigated.

\section{A. Primary parameters based on our model}

For modelling purposes in our LTI power line generator, the cable type is regarded as a two-conductor plus reference wire transmission line, with surrounding dielectric material of relative dielectric constant $\varepsilon_{r}$ - this assumption gives the following distributed parameters [14]:

$$
\begin{gathered}
R^{\prime}=\sqrt{\frac{\mu_{r} \mu_{0} f}{\pi \sigma a^{2}}}\left[\frac{\frac{d}{2 a}}{\sqrt{\left(\frac{d}{2 a}\right)^{2}-1}}\right] \\
L_{e x}=\frac{\mu_{r} \mu_{0}}{\pi} \cosh ^{-1}\left(\frac{d}{2 a}\right) ; L_{\text {in }}=\frac{R}{2 \pi f} ; L^{\prime}=L_{i n}+L_{e x} \\
C^{\prime}=\frac{\pi \varepsilon_{r} \varepsilon_{0}}{\cosh ^{-1}\left(\frac{d}{2 a}\right)} \\
G^{\prime}=2 \pi f C^{\prime} \tan \delta
\end{gathered}
$$

where $f$ is the wave frequency, $\sigma$ is the conductivity of material, $a$ is the conductor radius, $d$ is the distance between the centres of phase and neutral conductors, $\tan \delta$ is its dissipation factor, $\varepsilon_{0}$ is the permittivity of vacuum, $\mu_{0}$ represents free space permeability, and $\mu_{r}$ is its relative magnetic permeability [14], [15].

For further simulation and analysis we consider this calculation of primary parameters as calculation no. 1 . 


\section{B. Primary parameter based on FTW}

In the FTW PLC simulator, the transmission line parameters $R^{\prime}, L^{\prime}, G^{\prime}$, and $C^{\prime}$ were derived from the data provided by typical electrical-cable manufacturers. It is possible to choose from among five cable types with crosssections from $1.5 \mathrm{~mm}$ up to $10 \mathrm{~mm}$, but usually a cable cross-section greater than $4 \mathrm{~mm}$ is not used for in-home connections.

The resistance $R^{\prime}$ and conductance $G^{\prime}$ are frequencyselective and are given by:

$$
\begin{gathered}
R^{\prime}=R_{0} \cdot 10^{-5} \cdot \sqrt{f} \\
G^{\prime}=G_{0} \cdot 10^{-14} \cdot 2 \pi f
\end{gathered}
$$

where $f$ represents the frequency in [MHz]. The values $R_{0}$ and $G_{0}$ are constant for different cables depending on the size of the cross-section in $\mathrm{mm}^{2}$.

$C^{\prime}$ and $L^{\prime}$ are frequency invariant and also constant for different cables depending on the size of cross-section in $\mathrm{mm}^{2}$. The values of these primary parameters can be found in [11] or [12].

The Cañete PLC simulator furthermore takes into consideration the correction factor for conductance $G$ ' to overestimate cable losses [12].

For further simulation and analysis we consider this calculation of primary parameters as calculation no. 2 .

\section{Comparison of the calculations of primary parameters}

The analysis and comparison of the methods for primary parameter calculation show: a) the influence of the distance between the centres of phase and neutral conductors on primary parameters, b) the influence of frequency on primary parameters, and c) the influence of physical properties and material composition of the cable on primary parameters.
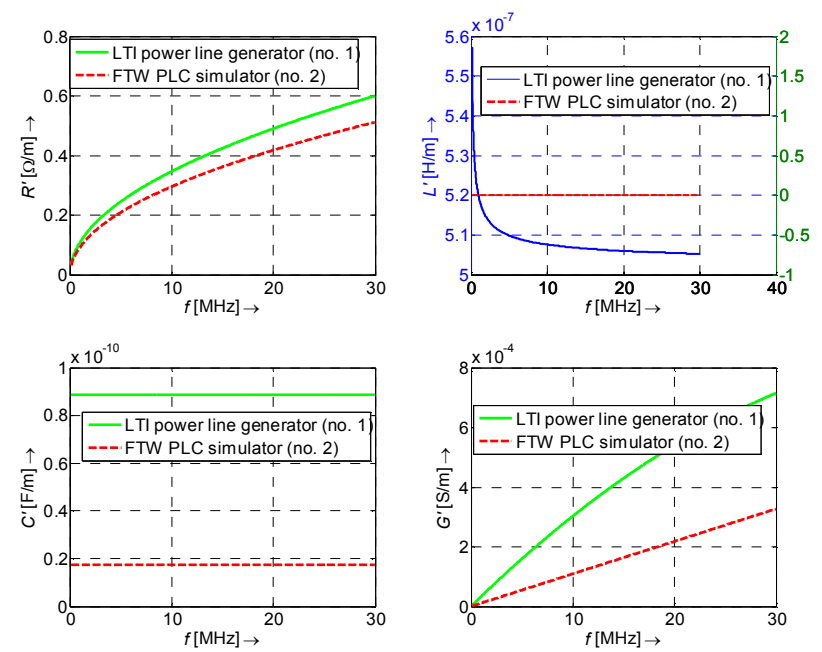

Fig.1. Comparison of the calculations of primary parameters.

Fig.1. shows the comparison of both approaches of the primary parameter calculation. The cable with the crosssection of $2.5 \mathrm{~mm}^{2}$ was considered. For this type of cable, the FTW PLC simulator and Cañete PLC simulator take into consideration the inductance $L^{\prime}=0.96 \mu \mathrm{H} / \mathrm{m}$ and the capacitance $C^{\prime}=17.5 \mathrm{pF} / \mathrm{m}$. Based on the different calculation of primary parameters, the cable of the same dimension shows different behaviour in the frequency range from 0 to $30 \mathrm{MHz}$.

Based on the primary parameters, the propagation constant $\gamma$ and the characteristic impedance $Z_{C}$ (secondary parameters) are computed using the equation provided, for example, in [16]. Fig.2. shows the results of the comparison of secondary parameters.
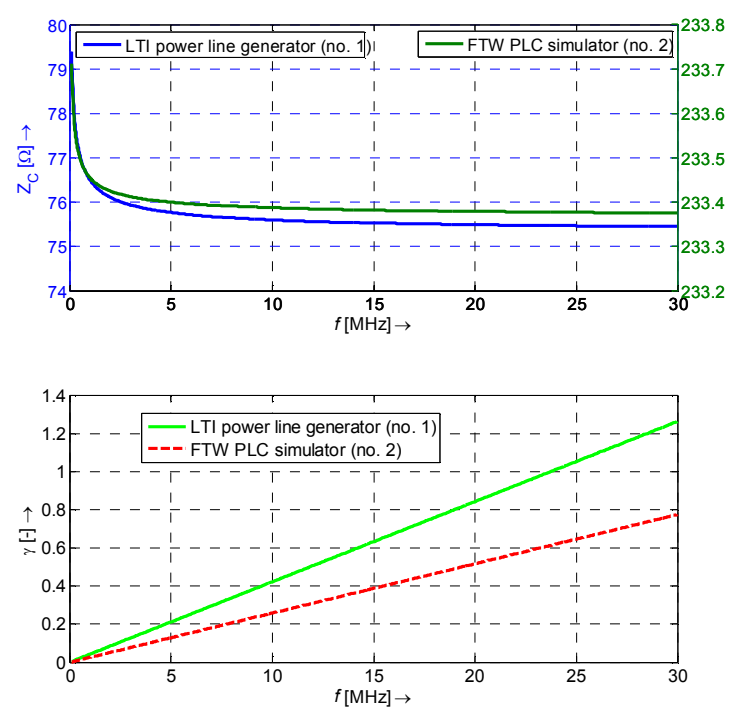

Fig.2. Comparison of secondary parameters.

Based on differences in the calculation of primary parameters, the characteristic impedance is three times higher for the FTW PLC simulator. The results of the measurements, published in [9] and [17], show the value of characteristic impedance $80-90 \Omega$, which corresponded with our model. On the other hand, in [18] the value $200 \Omega$ was introduced for characteristic impedance, which corresponded with the FTW PLC model.

The primary and secondary parameters are important input data for the power line modelling systems. It is therefore necessary to obtain accurate values, because cables which are not terminated with their characteristic impedance cause reflections and damping at some frequencies in the network. Reflections are caused by any difference in the characteristic impedance values along the line which may be generated by a variation in its dimensional parameters or by an unmatched load in the line.

\section{COMPARISON OF SIMULATORS AND ANALYSIS OF RESULTS}

The influence of different methods for the calculation of primary parameters on the transfer function was investigated.

Based on a topology considered in the FTW PLC simulator [11] and the Cañete PLC simulator [12], the simple fixed topology with three branches shown in Fig.3. and Fig.4. was considered for a comparison and investigation of the transfer function - the power line channel frequency response. 


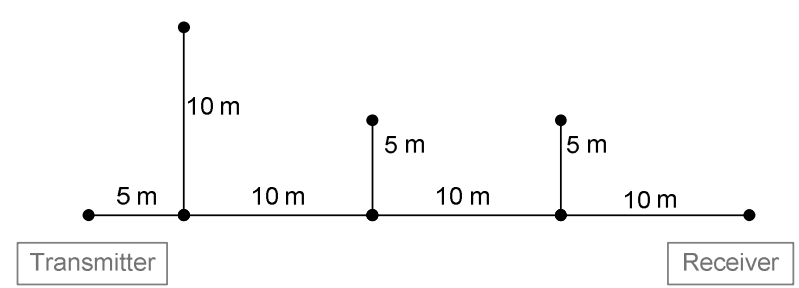

Fig.3. Proposed network topology based on Cañete topology [12].

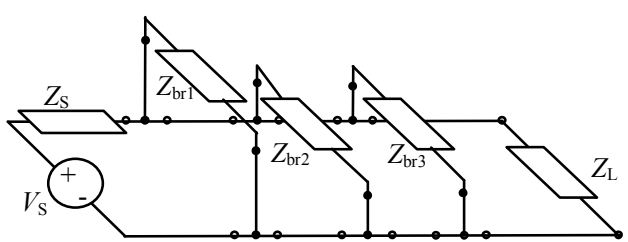

Fig.4. Proposed network topology $\left(Z_{b r l}=Z_{b r 2}=Z_{b r 3}=1000 \Omega, Z_{S}=Z_{L}=100 \Omega\right)$.

\section{A. Transfer function calculations}

The transfer function of the power line channel can be calculated by three possible approaches:

First, according to the description in [19] it is possible to calculate the transfer function as ratio between load voltage and source voltage using the equation (see Fig.5.):

$$
H=\frac{V_{L}}{V_{S}}=\left|\frac{Z_{C}}{A Z_{C}+B+C Z_{C} Z_{S}+D Z_{S}}\right|
$$

$A, B, C$, and $D$ are frequency dependent coefficients that are calculated from the secondary parameters: characteristic impedance $Z_{C}$ and propagation constant $\gamma$.

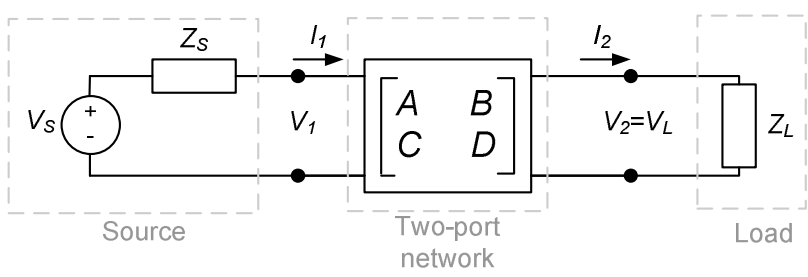

Fig.5. Two port network model.

Second, based on literature [20] and a detailed description in [11] it is possible to calculate the transfer function as ratio of power across the load $Z_{L}$ before and after the insertion of the channel, using the equation:

$$
I L=10 \log _{10} \frac{P_{\text {bef }}}{P_{\text {aft }}}=20 \log _{10}\left|\frac{V_{L 1}}{V_{L 2}}\right|=\left|\frac{Z_{L}+Z_{S}}{A Z_{L}+B+C Z_{L} Z_{S}+D Z_{S}}\right|
$$

Third, if we consider only the voltage ratio between the output and the input of a two-port network, the transfer function can be calculated using the equation:

$$
H_{\text {loop }}=\frac{V_{2}}{V_{1}}=20 \log _{10}\left|\frac{Z_{L} \cdot C+D}{Z_{L} \cdot A \cdot C+B \cdot C+A \cdot D+\frac{B \cdot D}{Z_{L}}}\right|
$$

\section{B. Comparison of transfer functions}

Fig.6. shows a comparison of the transfer functions $H_{\text {loop }}$ for our LTI power line generator and the FTW PLC simulator. The comparison shows significant differences between the transfer functions. Therefore, Fig.7. shows a comparison of transfer functions if the same approach of the calculation of primary parameters is considered. These results show the big influences of primary parameter calculation on the resulting transfer function. Fig.7. shows also a comparison with the Cañete PLC simulator. The compliance of transfer functions of our LTI power line generator and the Cañete PLC simulator in Fig.7. shows an exact match and confirms the correctness of the LTI power line generator design.

Fig.8. illustrates the comparison of the transfer function calculation from equations (7) - (9). The differences can be caused by the calculation of input impedance; the following section will therefore focus on the comparison of input impedances.

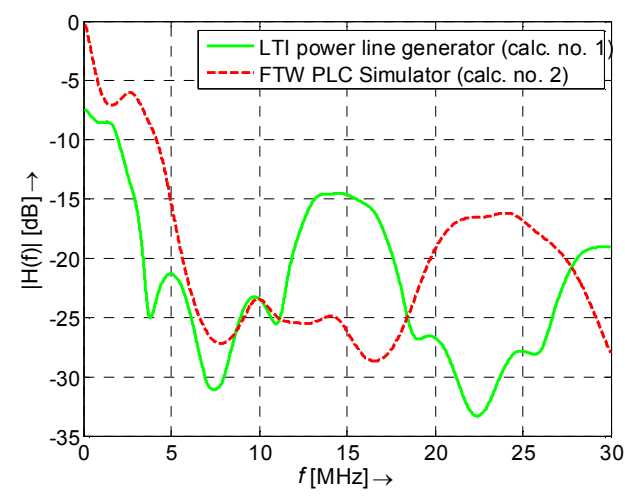

Fig. 6. Comparison of transfer function with the different primary parameter calculation.

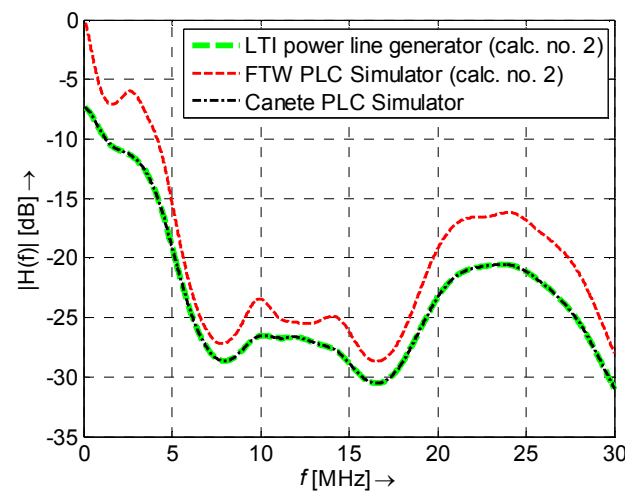

Fig.7. Comparison of transfer functions with the same primary parameter calculation and with Cañete PLC Simulator. 


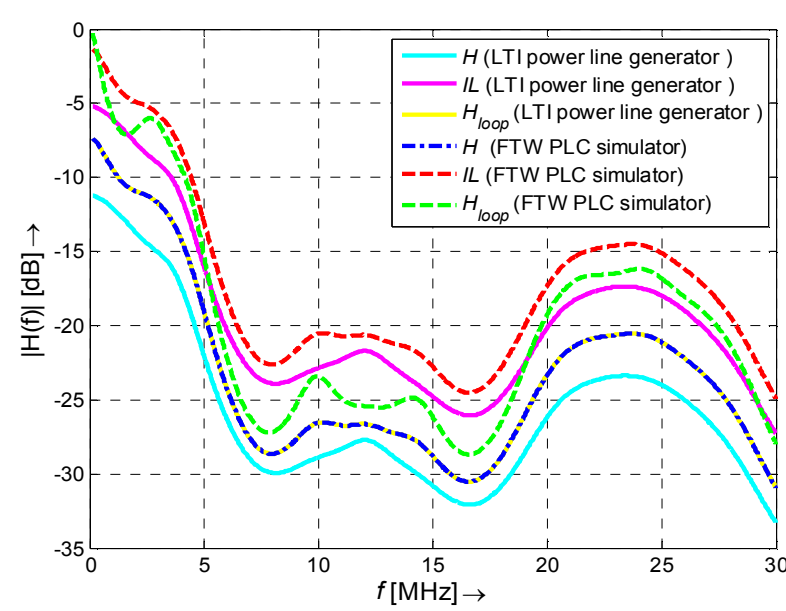

Fig.8. Comparison of different calculations of transfer function with the same primary parameter calculation (no. 2.).

\section{Comparison of input impedances}

The input impedance can also be calculated using the frequency dependent coefficients $A, B, C$, and $D$ and the load impedance using the equation [21]:

$$
Z_{I N}=\frac{A \cdot Z_{L}+B}{C Z_{L}+D}
$$

Fig.9. illustrates a comparison of the input impedance of our model and that of the FTW PLC simulator. From this figure we can observe that the differences between the input impedances are negligible, but this small difference caused a difference in the frequency response of the transfer function (see Fig.7.).

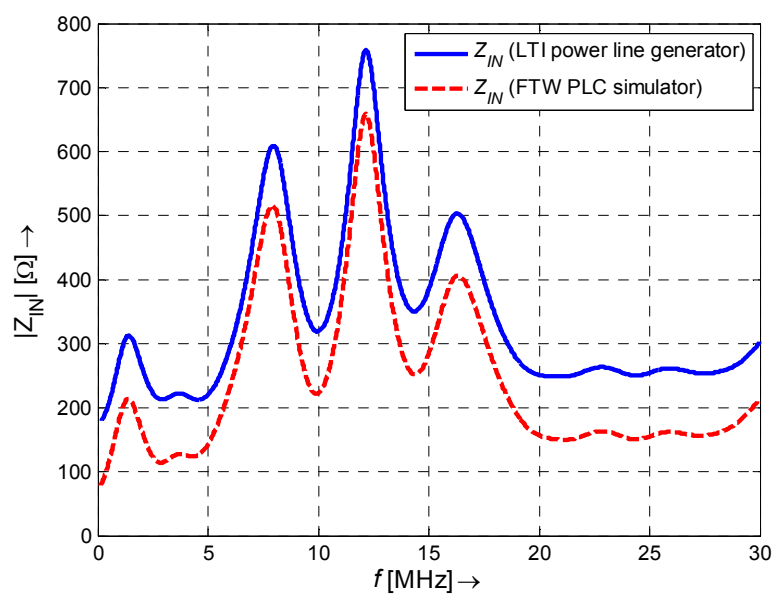

Fig. 9. Comparison of input impedances.

\section{Comparison of cable type}

Fig.10. shows the influence of cables with different dimensions on the transfer function. From Fig.10. we can see that the cables with a higher cross-section have a lower attenuation. The comparison shows negligible differences between cables with 1.5 and $2.5 \mathrm{~mm}^{2}$ cross-sections.

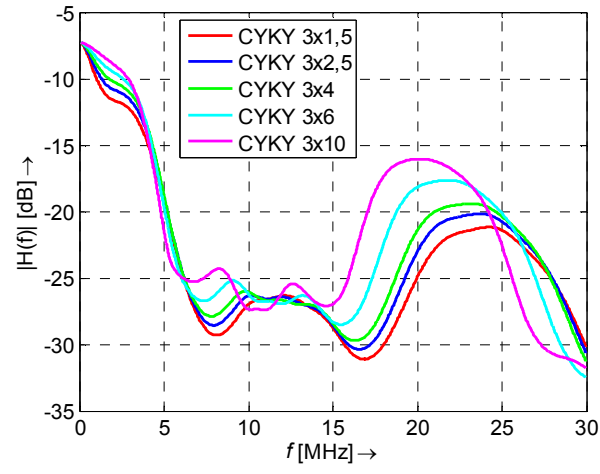

Fig.10. Comparison of transfer functions of power line cables with different dimensions.

\section{COMPARISON OF MEASUREMENTS}

The simple topology with one branch was considered for the measurement issue. Fig.11. shows the simple topology. The frequency response was measured using the Instek GSP-830 spectral analyser. Frequencies up to $100 \mathrm{MHz}$ were considered for the measurements. The frequencies up to $100 \mathrm{MHz}$ enable providing an analysis that covers the full bandwidth as defined in the G.hn standard [26].

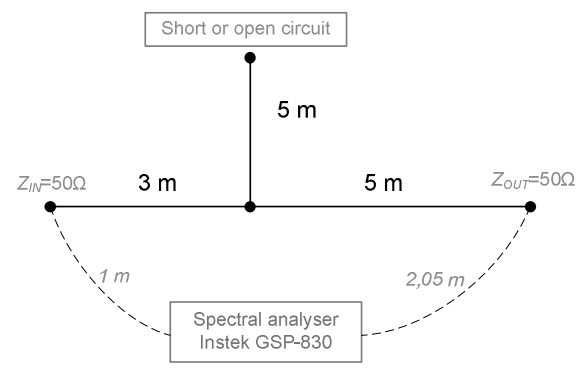

Fig.11. Simple topology for measurement.

Fig.12. shows a comparison of our LTI generator with no. 1 calculation of primary parameters and the FTW simulator with no. 2 calculation of primary parameters and measurement by the analyser. The short circuit impedance was considered for branch termination. Fig.13. shows the same comparison as Fig.12., but the open circuit was taken into consideration for branch termination.

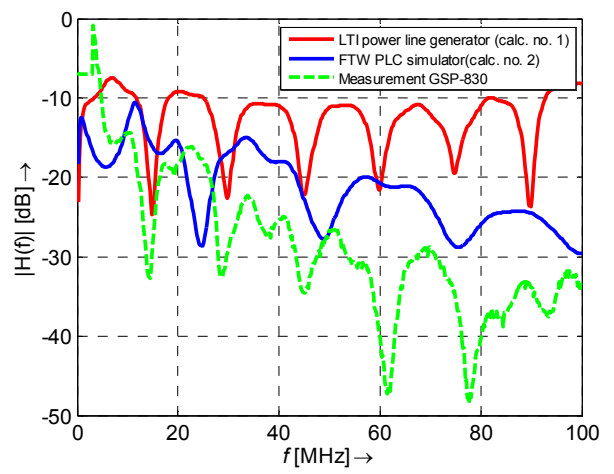

Fig.12. Comparison of models with measurement for topology with one branch terminated by short circuit impedance. 


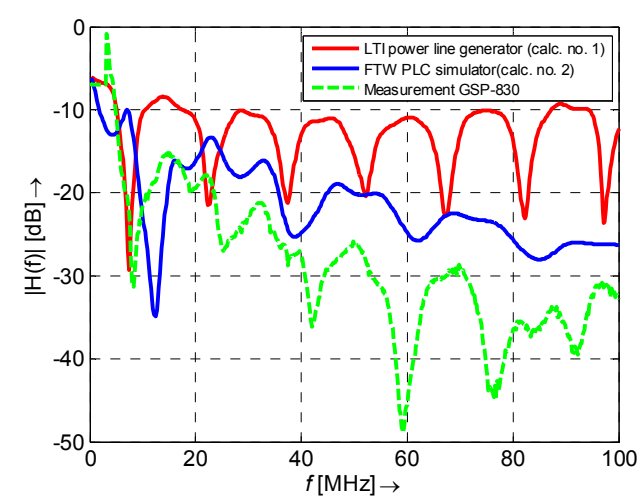

Fig.13. Comparison of models with measurement for topology with one branch terminated by open circuit impedance.

\section{Results of measurements - analysis and discussion}

\section{Notch position}

From the comparison in Fig.12. and considering only the LTI generator and the measurement results, the agreement in notch positions on frequencies around 15,30 , and $45 \mathrm{MHz}$ is obvious.

The numerical analysis validated the comparison results too. Due to the insulating material with the dielectric constant $\varepsilon_{r} \approx 4$, the phase speed on the cable is approximately $150 \mathrm{~m} / \mu \mathrm{s}$. The first peak in Fig. 12 . is the transmission impulse from the transmitter, which has traversed an entire cable length of $11.05 \mathrm{~m}$, thus appearing after a time of $11.05 / 150 \cdot 10^{6}=73.7 \mathrm{~ns}$. The second peak, originating from the fact that the signal passes through the tap and is reflected at the tap's open end, traversed a total length of $26.05 \mathrm{~m}$ appearing after $26.05 / 150 \cdot 10^{6}=140.3 \mathrm{~ns}$. A tap passage of $10 \mathrm{~m}$ takes about $66.6 \mathrm{~ns}$.

The effects of reflection in the tap appear in the transfer function (frequency response) in the form of notches with fixed frequency spacing. The first notch occurs where the direct and the reflected waves are shifted exactly a half wavelength against each other, which leads to subtraction. The first frequency $f_{1}=15 \mathrm{MHz}$ belonging to the first notch means that we have a period of $66.6 \mathrm{~ns}$. The repetition of notches occurs at multiples of the first frequency $f_{1}$.

\section{Attenuation}

The values of attenuation are significantly smaller for models than for the measurement. The behaviour of the frequency response of our LTI generator on frequencies higher than $50 \mathrm{MHz}$ is influenced by the conductance $G^{\prime}$. In Fig.14. the frequency characteristics of conductance $G^{\prime}$ and the decreasing values after $50 \mathrm{MHz}$ are shown. This decrease is caused by the dissipation factor $\tan \delta$ from equation (4).

The differences between the frequency response of the FTW simulator and the measurement can be caused by the relative dielectric constant $\varepsilon_{r}$, which is not considered in the calculation of primary parameters in the FTW simulator.

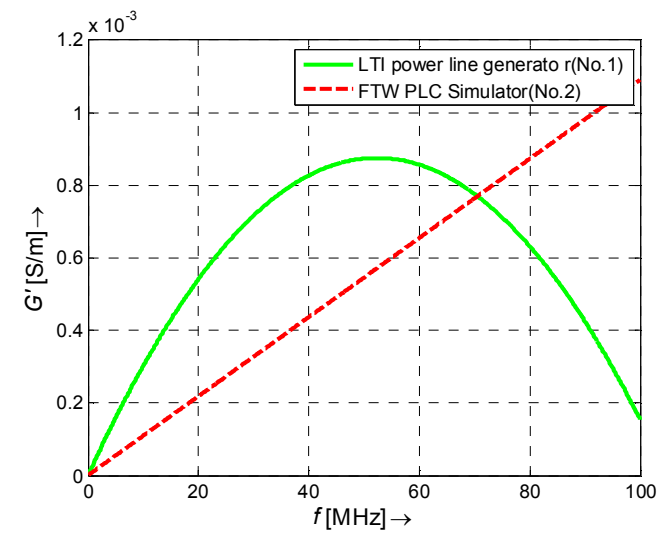

Fig.14. Conductance $G^{\prime}$ in frequency range up to $100 \mathrm{MHz}$.

\section{REAL-TIME ESTIMATION OF POWER LINE TRANSMISSION PARAMETERS}

The article shows that transmission line parameters are important input data for power line system modelling or other relevant applications. In current practice as was described in chapter 5, the transmission parameters are calculated using theoretically derived formulas that are based on the information about their size, length, structure, and type. But obviously, there is a difference between the calculated and the actual parameters because the transmission line parameters $R^{\prime}, L^{\prime}, G^{\prime}$, and $C^{\prime}$ vary in real conditions with the environment and weather (e.g. temperature and wind speed [22], [23]). Some methods for measuring the resistance and reactance of power lines were introduced in [24] and [25].

The assumption of time invariant transmission line parameters can lead to inaccuracy or erroneous results in modelling and system analysis. Chapter 6.B shows the significant influence of transmission power line parameters on the transfer function.

The analysis and experimental evaluation of power line transmission parameters carried out in this article create a foundation for the implementation of a method for real-time estimation of transmission line parameters. Real-time estimation of transmission line parameters is essential to enhancing accuracy in Smart Grid or PLC applications.

\section{CONCLUSION}

The proposed LTI power line generator for indoor power line communication was compared with the publicly available simulators. The comparison shows the huge influence of the primary parameter calculation, and therefore an analysis of two primary parameter calculation approaches was conducted too. For both types of calculation there is literature available which considers the first or the second type of calculation. The article presented a detailed comparison of both calculation approaches. The measurement comparison shows more accurate results in notch positions for no. 1 calculation used in the proposed LTI power line generator. On the other hand, the comparison with the Cañete PLC channel generator shows better results for no. 2 calculation used in the FTW PLC simulator. 
In the case of comparing the proposed LTI generator and the FTW simulator with the same calculation of primary parameters, the differences are probably caused by the input impedance. The input impedance is the most significant parameter affecting the shape of the transfer function.

The measurements were conducted for frequencies up to $100 \mathrm{MHz}$ and the notch positions and attenuation were analysed and discussed. Frequencies up to $100 \mathrm{MHz}$ need to be considered to cover the full bandwidth, as was defined in the broadband PLC standards ITU-T G.hn [26] and IEEE 1901 [27].

The proposed generator and analysis of parameters which significantly influenced the transfer function could be used for a method for managing power line topology. Based on the knowledge of the transfer function, the communication parameters such as modulation, code rate, power level, amplification, sub carriers or coding could be changed. This issue becomes extremely important and necessary nowadays. For example, present-day PLC standards use adaptive tone mapping, which adaptively selects optimum modulation [28].

The analysis, the PLC generator and simulators are also suitable for investigating some subchannels with deep fading conditions, which make these subchannels unsuitable for data transmission, especially in the case of Orthogonal Frequency-Division Multiplexing systems used for the indoor power line specified in the ITU G.hn and IEEE 1901.

\section{ACKNOWLEDGMENT}

Research described in this article was financed by the National Sustainability Program under grant LO1401 and by the Czech Science Foundation under grant no. GP1429084P. For the research, infrastructure of the SIX Center was used.

\section{REFERENCES}

[1] Dostert, K. (1997). Telecommunications over the power distribution grid - possibilities and limitations. In International Symposium on Power-Line Communications and Its Applications, April 1997. Essen, Germany, 1-9.

[2] Ferreira, H., Lampe, L., Newbury, J., Swart, T. (2010) Power Line Communications, (1st ed.). Wiley.

[3] Galli, S., Scaglione, A., Wang, Z. (2011). For the grid and through the grid: The role of power line communications in the smart grid. Proceedings of the IEEE, 99 (6), 998-1027.

[4] Ferreira, H., Lampe, L., Newbury, J., Swart, T. (2010). Power Line Communications: Theory and Applications for Narrowband and Broadband Over Power Lines, (1st ed.). Wiley.

[5] Zimmermann, M., Dostert, K. (2002). A multipath model for the powerline channel. IEEE Transactions on Communications, 50 (4), 553-559.

[6] Galli, S., Banwell, A. (2005). A novel approach to accurate modeling of the indoor power line channelPart II: Transfer function and channel properties. IEEE Transactions on Power Delivery, 20 (3), 1869-78.
[7] Cañete, F., Cortes, J., Diez, L., Entrambasaguas, J. (2006). Analysis of the cyclic short-term variation of indoor power line channels. IEEE Journal on Selected Areas in Communications, 24 (7), 1327-1338.

[8] Tonello, A., Versolatto, F. (2009). New results on topdown and bottom-up statistical PLC channel modeling. In Third Workshop on Power Line Communications, 1-2 October 2009. Udine, Italy, 1-4.

[9] Mlynek, P., Misurec, J., Koutny, M. (2013). Random channel generator for indoor power line communication. Measurement Science Review, 13 (4), 206-213.

[10] Marrocco, G., Statovci, D., Wolkerstorfer, M. (2014). FTW PLC Simulator [computer software]. Wien, Austria.

[11] Marrocco, G., Statovci, D., Trautmann, S. (2013). A PLC broadband channel simulator for indoor communications. In 17th IEEE International Symposium on Power Line Communications and Its Applications, 24-27 March 2013. IEEE, 321-326.

[12] Cañete, F., Cortes, J., Diez, L., Entrambasaguas, J. (2011). A channel model proposal for indoor power line communications. IEEE Communications Magazine, 49 (12), 166-174.

[13] Mlynek, P., Misurec, J., Koutny, M. (2013). Hybrid power line model based on frequency and time-variant system. In Global Information Infrastructure Symposium, 28-31 October, 2013. IEEE, 1-3.

[14] Papaleonidopoulos, I.C., Karagiannopoulos, C.G., Theodorou, N.J., Anagnostopoulos, C.E., Anagnostopoulos, I.E. (2002). Modelling of indoor low voltage power-line cables in the high frequency range. In International Symposium on Power Line Communications and Its Applications, March 27-29, 2002. Athens, Greece, 267-271.

[15] Meng, H., Chen, S., Guan, Y.L., Law, C.L., So, P.L., Gunawan, E., Lie, T.T. (2002). A transmission line model for high-frequency power line communication channel. In International Conference on Power System Technology (PowerCon 2002). IEEE, Vol. 2, 12901295.

[16] Zimmermann, M., Dostert, K. (1999). A multi-path signal propagation model for the power line channel in the high frequency range. In 3rd International Symposium on Power-Line Communications. Lancaster, UK, 45-51.

[17] Yazdani, J., Naderi, M., Honary, B. (2001). Power lines analysing tool (PLAT) for channel modelling. In 5th International Symposium on Power-Line Communications and Its Applications, 4 April 2001. Malmö, Sweden, 27-28.

[18] Cañete, F., Cortes, J., Diez, L., Entrambasaguas, J. (2003). Modeling and evaluation of the indoor power line transmission medium. IEEE Communications Magazine, 41 (4), 41-47.

[19] Mlynek, P., Misurec, J., Koutny, M., Silhavy, P. (2012). Two-port network transfer function for power line topology modeling. Radioengineering, 21 (1), 356-363. 
[20] Golden, P., Dedieu, H., Jacobsen, K.S. (2004). Fundamentals of DSL Technology, (1st ed.). Auerbach Publications.

[21] Banwell, T.C., Galli, S. (2001). On the symmetry of the power line channel. In 5th International Symposium on Power Line Communications and Its Applications, 4 April 2001. Malmö, Sweden, 325-330.

[22] Electric Power Research Institute. (2006). EPRI AC Transmission Line Reference Book - $200 \mathrm{kV}$ and Above, (3rd ed.). Palo Alto, CA: EPRI.

[23] Douglass, D.A., Kirkpatrick, L.A. (1985). AC resistance of ACSR-magnetic and temperature effects. IEEE Transactions on Power Apparatus and Systems, PAS-104 (6), 1578-1584.

[24] Allfather, L.A. (1998). Impedance measuring. U.S. Patent No. 5818245. Washington, D.C.: U.S. Patent and Trademark Office.
[25] Bachmann, B., Hart, D.G., Hu, Y., Novosel, D., Saha, M.M. (2002) Impedance measurement system for power system transmission lines. U.S. Patent No. 6397156. Washington, D.C.: U.S. Patent and Trademark Office.

[26] International Telecommunication Union. (2011). Unified high-speed wire-line based home networking transceivers-system architecture and physical layer specification. ITU-T Recommendation G.9960.

[27] IEEE Standards. (2010). Standard for Broadband over Power Line Networks: Medium Access Control and Physical Layer Specifications. IEEE Std 1901-2010.

[28] Han Kim, Il., Dabak, A.G., Pande, T. (2013). Adaptive tone power control in plc networks. U.S. Patent No. 20130089124 A1. Washington, D.C.: U.S. Patent and Trademark Office. 